\title{
PROSPECTIVE STUDY ON THE EFFECT OF TOREMIFENE IN PATIENTS WITH ADJUVANT ANASTROZOLE FAILURE IN POSTMENOPAUSAL BREAST CANCER
}

\author{
Reiki Nishimura $^{1)}$, Seigo Nakamura ${ }^{2)}$, Koji Oba ${ }^{3)}$, Yoshifumi Komoike ${ }^{4)}$, Shoshu Mitsuyama ${ }^{5)}$, \\ Kazufumi Hisamatsu ${ }^{6}$, Noboru Oriuchi ${ }^{7}$, Chigusa Abe ${ }^{8)}$, Takashi Kuwayama, ${ }^{90}$, Tetsuya Taguchi ${ }^{11)}$, \\ Junichi Sakamoto ${ }^{12)}$ and Shinzaburo Noguchi ${ }^{13)}$ \\ ${ }^{1)}$ Department of Breast and Endocrine Surgery, Kumamoto City Hospital, ${ }^{2)}$ Department of Breast Surgical Oncology, Showa \\ University School of Medicine, ${ }^{3)}$ Translational Research and Clinical Trial Center, Hokkaido University Hospital, Hokkaido \\ University, ${ }^{4)}$ Department of Breast and Endocrine Surgery, Osaka Medical Center for Cancer and Cardiovascular Diseases, \\ ${ }^{5)}$ Department of Surgery, Kitakyushu Municipal Medical Center, ${ }^{6)}$ Department of Surgery, Hiroshima City Asa Hospital, \\ ${ }^{7)}$ Department of Diagnostic Radiology and Nuclear Medicine, Gunma University Graduate School of Medicine, \\ ${ }^{8)}$ Epidemiological \& Clinical Research Information Network, ${ }^{9)}$ Department of Breast Surgical Oncology, St.Luke's \\ International Hospital, ${ }^{10)}$ Department of Breast Surgical Oncology, Tokyo Medical and Dental University, \\ ${ }^{11)}$ Department of Endocrine and Breast Surgery, Kyoto prefectural University of Medicine, ${ }^{12)}$ Young Leaders' Program in \\ Healthcare Administration, Nagoya University Graduate School of Medicine, ${ }^{13)}$ Department of Breast and Endocrine Surgery, \\ Osaka University Graduate School of Medicine
}

\begin{abstract}
Purpose; An endocrine treatment using aromatase inhibitor (AI) is a standard strategy for postmenopausal patients with hormone receptor positive breast cancer. Postmenopausal patients who relapsed during adjuvant AI treatment or progression within a year after completion of AI treatment were prospectively treated with $40 \mathrm{mg}$ /day of toremifene (TOR) in order to examine the effect of the selective estrogen receptor modulator (SERM) in AI-refractory patients.

Patients and Methods; Twenty-three postmenopausal breast cancer patients who relapsed during adjuvant AI treatment or relapsed within a year after completion of AI were enrolled in the prospective trial from January 2007 to February 2010. Out of the patients, 14 cases had measurable or evaluable lesions, and the other had only bone metastasis. All patients were treated with toremifene of $40 \mathrm{mg} /$ day. The primary efficacy end point for the trial was clinical response and secondary end point was progression free survival (PFS).

Result; The objective response rate in the patients with measurable lesions was $7.1 \%(1 / 14)$ and clinical benefit rate was $28.6 \%$. In the patients with evaluable bone disease, clinical benefit was $44.4 \%$. No serious adverse event was observed except a patient with grade 3 non-hematological toxicity (AST and ALT).

Conclusion; The SERM, toremifene (40 mg/day), demonstrated a safe profile and a favorable effect on disease control after adjuvant AI failure.
\end{abstract}

Key Words: toremifene, adjuvant anastrozole failure, prospective study, post menopausal breast cancer

(Received February 5, 2011; Accepted February 9, 2011)

\section{Introduction}

An adjuvant treatment strategy incorporating an aromatase inhibitor (AI) reduces the risk of breast cancer recurrence compared with 5 years of tamoxifen (TAM) alone ${ }^{1,2)}$. American Society of Clinical Oncology Clinical Practice Guideline recommends that postmenopausal women with hormone receptor-positive breast cancer consider incorporating AI therapy at some point during adjuvant treatment, either as up-front therapy or

Correspondence and reprint request to: Junichi Sakamoto MD, PhD, FACS. Department of Social Life Science, Nagoya University Graduate School of Medicine 65 Tsurumai-cho, Showa-ku, Nagoya 466-8550, Japan. TEL: +81-52-744-2442, email: sakamjun@med.nagoya-u.ac.jp as sequential treatment after $\mathrm{TAM}^{3)}$. The optimal timing and duration of endocrine treatment remain unresolved. The Update Committee supports careful consideration of adverse effect profiles and patient preferences in deciding whether and when to incorporate AI therapy. Thus, AI is shown to be one of the effective endocrine treatments for postmenopausal patients. AI is divided into two types, i.e. steroidal and non-steroidal, and has three medications; anastrozole, exemestane, and letrozole. In recurrent breast cancer, AI had been used as a second line endocrine treatment after failure on TAM. According to the several pooled studies, these three agents have been recommended as the first line treatment for advanced or recurrent disease, and also as adjuvant therapy in postmenopausal patients ${ }^{3,4)}$. 
Toremifene (TOR) is a selective estrogen receptor modulator (SERM), and it was reported that the efficacy in postmenopausal breast cancer patients was similar to that of $\mathrm{TAM}^{5)}$. TOR was reported to compete with estrogen at the site of the estrogen receptor (ER) and to suppress insulin-like growth factor-I-dependent growth ${ }^{6}$. Additionally, it has non-ER dependent anti-tumor effect such as suppression of angiogenesis ${ }^{7}$.

In the present study, we prospectively registered patients with recurrent postmenopausal patients who relapsed during adjuvant AI treatment or progression within a year after completion of AI treatment. The patients were subsequently treated with TOR in order to investigate the effect of TOR for AI-refractory cases.

\section{Patients and Methods}

\section{Patients}

The patients enrolled in this study had either advanced or recurrent breast cancer, in which the primary lesion was histologically or cytologically found to be an invasive carcinoma. In addition, the patients met the following inclusion criteria: (1) postmenopausal patients who underwent total mastectomy or breast-conserving surgery , (2) recurrence during adjuvant AI treatment (duration of AI treatment $>2$ years), or (3) recurrence within a year after completion of adjuvant AI, (4) recurrent lesions evaluable by response evaluation criteria in solid tumors (RECIST) or bone metastasis confirmed by the criteria of 14th edition of Japan Breast Cancer Society, (5) ER and/or PgR positive, (6) a performance status (PS) of $0-2$, (7) with adequate hematological, hepatic, renal, and cardiac function.

Postmenopausal breast cancer patients who were refractory to adjuvant AI were defined eligible for this study. There was no case with TAM as an adjuvant therapy. Each patient was required to have measurable disease by CT imaging, or bone metastatic lesion which must have been confirmed by MRI. After registration to this study, TOR of $40 \mathrm{mg} /$ day was administered and continued until treatment failure or disease progression. The case report form was used to collect information on each patient, including data on clinicopathological factors at the time of the initial operation, time to recurrence, sites of recurrence. The ethics of the study were approved by the institutional review board of participating institutions.

The registration of each patient was performed by sending the case report form for evaluation of eligibility criteria at ECRIN data center.

\section{Methods}

The primary endpoint was tumor response evaluated every 8 weeks based on response evaluation criteria in solid tumors (RECIST). The objective response rate was defined by the measurable lesions as complete and partial response $(\mathrm{CR}+\mathrm{PR})$, and the clinical benefit rate as the frequencies of $\mathrm{CR}+\mathrm{PR}+$ long stable disease (SD). PD was defined as an increase of at least $25 \%$ in the size of at least one bidimensionally or unidimensionally measurable lesion or as the appearance of any new lesion. Cases where metastatic lesions were present only in the bones were separately evaluated whether there is any exacerbation of bone metastasis (PD) or no change during TOR therapy (SD). The secondary end point for the trial was PFS, defined as the length of time between the administration of $\mathrm{AI}$ and the onset of disease progression or death. When progressive disease (PD) was established, the time to progression was the time from the beginning of treatment until the day on which progression was suspected and documented.

Toxicities were determined by the Common Terminology Criteria for Advance Events (CTCAE version 3.0).

The ER, PgR, and human epithelial growth factor 2 (HER2) statuses for each patient were measured. ER and $\mathrm{PgR}$ were evaluated by immunohistochemistry, and a value of $10 \%$ or higher was rated as positive. HER2 was assayed by immunohistochemistry or FISH. HER2positivity was indicated by $3+$ staining intensity. HER2equivocal $(2+$ staining) was tested using fluorescence in situ hybridization with the threshold for positive HER2:CEP17 ratio of $>2.0$.

\section{Statistical analysis}

For a two-sided $95 \%$ confidence interval (CI) for a binomial proportion whose true value is 0.1 , a sample size of 23 yields a half-width of at most 0.15 with a conditional probability of 0.807 . Continuous variables were compared using the Student's t test. Frequency analysis was performed with the $\chi 2$ test. Response rate and clinical benefit rate were shown with a two-sided 95\% Agresti-Coull CIs. Time to treatment failure was analyzed using the Kaplan-Meier method. All statistical analyses were performed using the SAS version 9.2 (SAS Institute Inc., Cary, NC).

\section{Results}

\section{Patients Characteristics}

Twenty four patients were registered to the study. One patient was considered ineligible because of the non-metastatic disease after meticulous audit. Baseline demographic characteristics of the remaining 23 patients are listed both for measurable disease (Table 1) and bone metastatic disease (Table 2) respectively. Fourteen patients were assessable for response using intent-to-treat analysis. Nine patients had only bone metastatic lesions and disease progression was assessed by MRI and/or 
Table 1. Baseline Characteristics of Patients with Measurable Disease

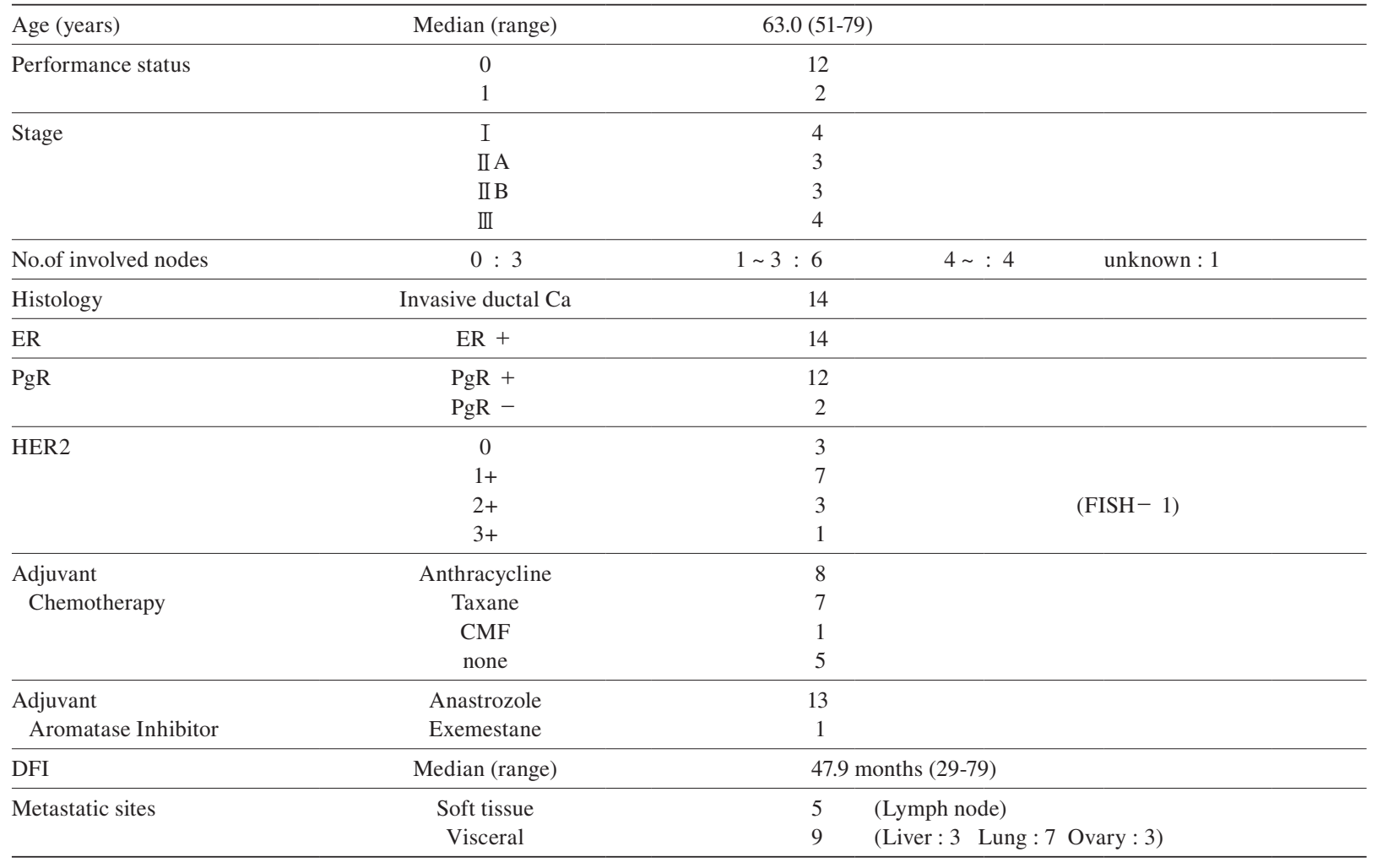

Table 2. Baseline Characteristics of the Patients with Metastatic Bone Disease

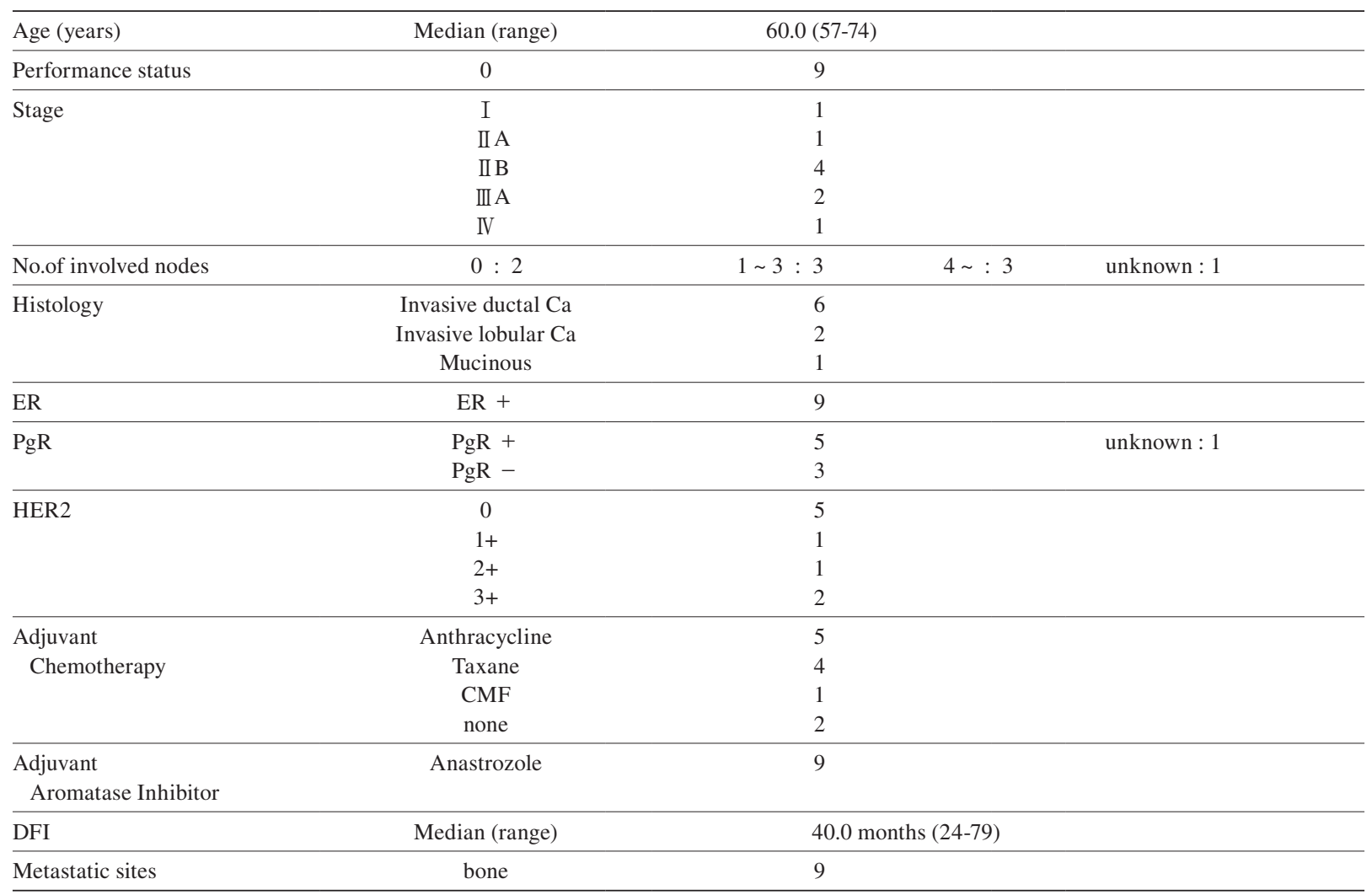


Table 3. Clinical Response in Patients with measurable disease

\begin{tabular}{lcl}
\hline Clinical Response & PR & 1 \\
& Long SD & 3 \\
& SD & 5 \\
& PD & 5 \\
\hline Progression-free survival & median (range) & 3.7 months $(0.7 \sim 14.7)$ \\
& & \\
\hline
\end{tabular}

Table 4. Clinical Response in Patients with Bone metastasis

\begin{tabular}{lll}
\hline Clinical Response & Long SD & 4 \\
& SD & 2 \\
& PD & 2 \\
& NA & 1 \\
\hline Progression-free survival & median (range) & \multirow{2}{*}{3.6 months $(0.9 \sim 19.6)$} \\
\hline
\end{tabular}

bone scintigraphy separately. All the patients had ER positive tumors, 12 out of the 14 patients were PgR positive among the measurable group, and 3 out of 8 patients (unknown in one case) with only bone metastasis group. Median disease-free interval (DFI) in patients with measurable disease was 47.9 months and that in patients with bone metastasis was 40.0 months. Metastatic sites were as follows; soft tissue in 5 cases, visceral in 9 cases and bone in 9 cases.

\section{Treatments}

All the patients had received curative operation between March 2002 and April 2007. Postoperative adjuvant endocrine therapy consisted of Anastrozole (ANA) in all patients except one who received exemestane therapy, and also various other additional chemotherapies were added in considerable numbers of the patients (Table 1 and 2).

\section{Outcome}

The objective response rate in patients with measurable disease was $7.1 \%(1 / 14 ; 95 \% \mathrm{CI}=0.0-33.9 \%)$ and disease control rate was $64.3 \%(9 / 14 ; 95 \% \mathrm{CI}=21.5$ - $67.4 \%$ ). In one patient, obvious partial response was observed and the lesion was stable over one year (Fig. 1, Table 3).

In the patients with evaluable bone disease, disease control rate was $66.7 \%(6 / 9 ; 95 \%, \mathrm{CI}=26.7-80.9 \%)$. The clinical benefit rates $(\mathrm{CB}=\mathrm{CR}+\mathrm{PR}+$ stable disease (SD) 24 weeks) were $28.6 \%$ and $44.4 \%$, respectively. No serious adverse event was observed except a patient with toxicity of grade 3 (AST and ALT).

The data collection for survival analysis was completed in November 2010. The Kaplan-Meier projected 18.4 weeks of median time to progression $(95 \% \mathrm{CI}=11.1$ to 35.0 weeks: Fig. 2). The median time to treatment failure was defined as the time elapsed from day 1 to the time of withdrawal from study for any primary reason for withdrawal except partial response or complete response. The median projected 18.3 weeks of time to treatment failure (95\% CI = 10.1 to 33.1 weeks: Fig. 3 ). At the time of this analysis, there was no death. Twenty patients withdrew from the study because of the progressive disease. One patient was taken off study because of drug-related adverse events (hepatic dysfunction). Two patients stopped treatment because of their preferences.

\section{Discussion}

AI is indicated for the treatment of early and advanced ER positive breast cancer in postmenopausal women. In this study, the efficacy of TOR was investigated in the patients who relapsed during AI adjuvant therapy or progression within a year after completion of AI treatment. The objective response rate in patients with measurable disease was $7.1 \%$ and disease control rate was $64.3 \%$. In the patients with bone disease, disease control rate was $66.7 \%$. Moreover, the clinical benefit rates were $28.6 \%$ and $44.4 \%$, respectively. According to the TARGET trial $^{8)}$, the response rate of TAM was $10 \%$ in patients with relapse after adjuvant ANA treatment. With regard to second-line treatment with TAM after first-line AI failure, $48.7 \%$ gained clinical benefit, and $10.1 \%$ had an objective response ( $\mathrm{OR}=\mathrm{CR}+\mathrm{PR})$. On the other hand, $56.8 \%$ gained $\mathrm{CB}$ and $7.4 \%$ had an OR in the patients with AI after first-line TAM failure.

In the cases with non-steroidal AI failure, there were some reports concerning the efficacy of steroidal AI and pure antiestrogen $^{9-11)}$. The OR was $4.2-10.0 \%$ and the clinical benefit rate was $20.0-40.0 \%$. Regarding TOR, a high dose TOR showed OR of $15 \%$ and CB rate of $45.0 \%$ in a retrospective study ${ }^{12)}$, and it is suggested that high 


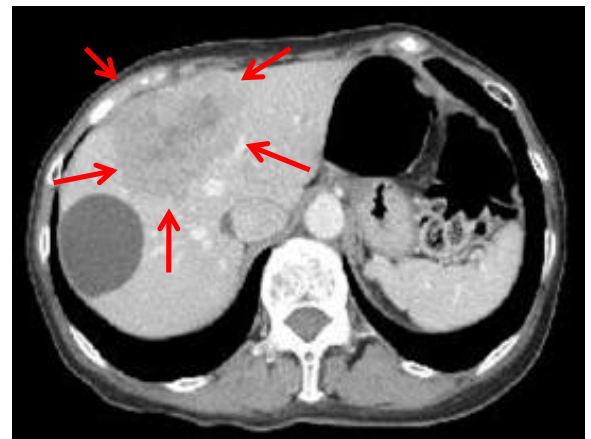

a) at the time of registration

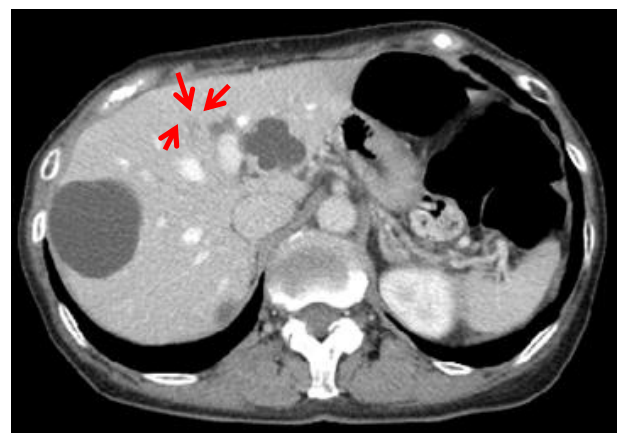

c) 1 year after treatment

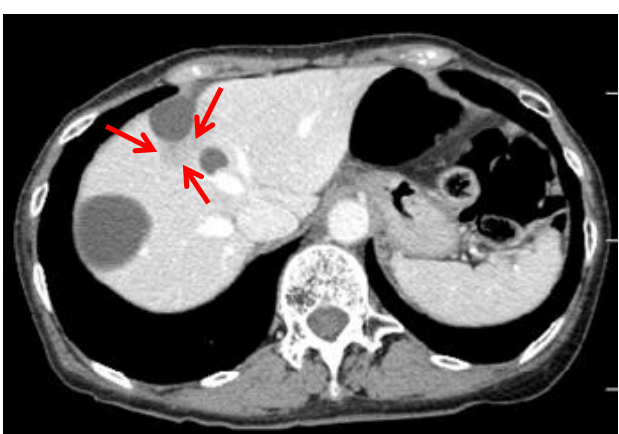

b) 3 month after treatment

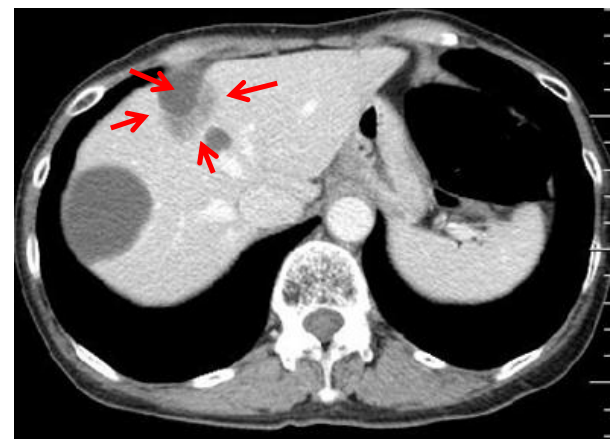

d) 20 months after treatment

Fig 1. Liver metastasis before and after toremifene treatment in a case who obtained partial response. Abdominal CT scan shows a huge liver metastasis (a). Fig.1-b shows a regression of the tumor and confirmed partial response and continued for over a year. Fig. 1-d shows a possible exacerbation of the metastatic site.

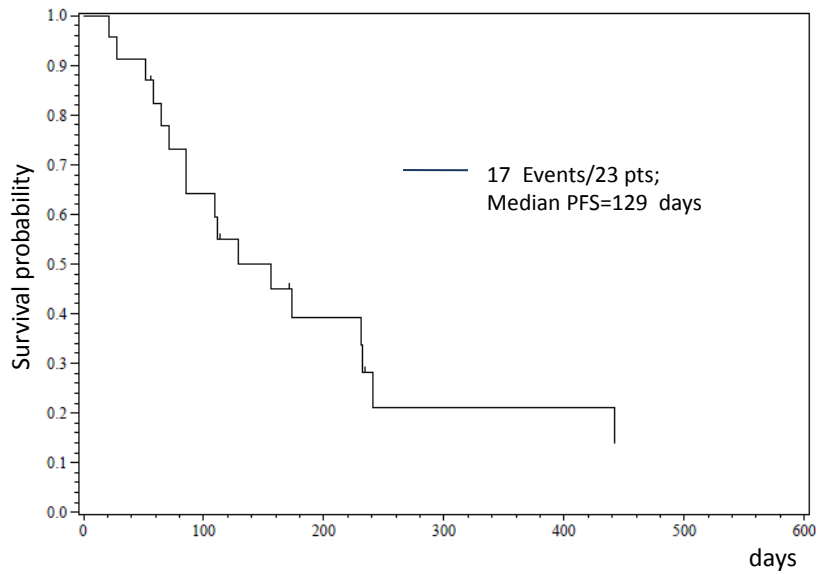

Fig 2. Kaplan-Meier curve of the total 23 patients enrolled into the study (Median Progression Free Survival Time).

dose TOR demonstrated the acceptability and efficacy. However, the efficacy of TOR in cases of AI adjuvant failure has not been evaluated in a systematic clinical trial. The present trial demonstrated that TOR at $40 \mathrm{mg} /$ day resulted in limited activity for patients with AI-refractory recurrent breast cancer. There are no comparable data for TOR $40 \mathrm{mg} /$ day versus high dose TOR. However, Osborne's review indicated that cross-resistance occur between TAM and TOR $40 \mathrm{mg} / \mathrm{day}^{13)}$. And high dose

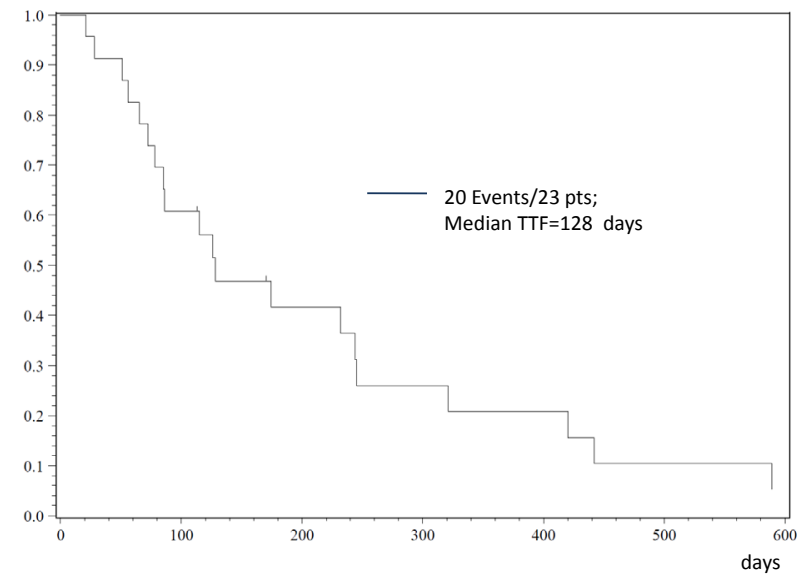

Fig 3. Kaplan-Meier curve of the total 23 patients enrolled into the study (Time to Treatment Failure).

TOR has shown efficacy in patients who progressed on $\mathrm{TAM}^{14,15)}$. There is a possibility that TOR $40 \mathrm{mg} /$ day is an effective treatment for AI adjuvant failure who did not received prior TAM treatment.

In conclusion, plausible response rate, disease control, and acceptable toxicity were proved, and TOR could be one of the promising treatment options for hormone-receptor positive postmenopausal breast cancer even after the adjuvant AI failures. 


\section{Acknowledgements}

This work is supported, in part, by a non-profit organization Epidemiological \& Clinical Research Information Network (ECRIN).

\section{References}

1) Cuzick, J., Sestak, I., Baum, M., Buzdar, A., Howell, A., Dowsett, M., Forbes, J.F. and ATAC/LATTE investigators. (2010) Effect of anastrozole and tamoxifen as adjuvant treatment for early-stage breast cancer: 10-year analysis of the ATAC trial. Lancet Oncol 11(12): 1135-41

2) Coates, A.S., Keshaviah, A., Thürlimann, B., Mouridsen, H., Mauriac, L., Forbes, J.F, Paridaens, R., Castiglione-Gertsch, M., Gelber, R.D., Colleoni, M., Láng, I., Del, Mastro, L., Smith, I. Chirgwin, J., Nogaret, J.M., Pienkowski, T., Wardley, A., Jakobsen, E.H., Price, K.N. and Goldhirsch, A. (2007) Five years of letrozole compared with tamoxifen as initial adjuvant therapy for postmenopausal women with endocrine-responsive early breast cancer: update of study BIG 1-98. J Clin Oncol. 25(5): 486-92.

3) Burstein, H.J., Prestrud, A.A., Seidenfeld, J., Anderson, H., Buchholz, T.A., Davidson, N.E., Gelmon, K.E., Giordano, S.H., Hudis, C.A., Malin, J., Mamounas, E.P., Rowden, D., Solky, A.J., Sowers, M.R., Stearns, V., Winer, E.P., Somerfield, M.R., Griggs, J.J.; American Society of Clinical Oncology. (2010) American Society of Clinical Oncology clinical practice guideline: update on adjuvant endocrine therapy for women with hormone receptorpositive breast cancer. J ClinOncol 28(23): 3784-96.

4) Mauri, D., Pavlidis, N., Polyzos, N.P. and Ioannidis, J.P. (2006) Survival with aromatase inhibitors and inactivators versus standard hormonal therapy in advanced breast cancer: meta-analysis. J Natl Cancer Inst 98(18): 1285-91.

5) Pyrhönen, S., Ellmén, J., Vuorinen, J., Gershanovich, M., Tominaga, T., Kaufmann, M. and Hayes, D.F. (1999) Meta-analysis of trials comparing toremifene with tamoxifen and factors predicting outcome of antiestrogen therapy in postmenopausal women with breast cancer. Breast Cancer Res Treat 56(2): 133-43.

6) Maruyama, S., Koga, K., Kuroiwa, S. and Ekimoto, H. (1993) Growth inhibitory effect of toremifene (NK622), a new antiestrogenic drug and its metabolite on human breast cancer cells in the presence of growth factor. J Jpn Soc Cancer Ther 28(5): 826-834.
7) Okada, M., Ogasawara, A., Sekine, K., Seno, C. and Nishikawa, K. (2001) Antiangiogenic and antimetastatic effects of toremifene citrate. Jpn J Cancer Chemother 28(8): 1099-1104.

8) Thürlimann, B., Robertson, J.F., Nabholtz, J.M., Buzdar, A., Bonneterre, J., Arimidex Study Group (2003) Efficacy of tamoxifen following anastrozole ('Arimidex') compared with anastrozole following tamoxifen as first-line treatment for advanced breast cancer in postmenopausal women. Eur J Cancer 39(16): 2310-7.

9) Lønning, P,E., Bajetta, E., Murray, R., Tubiana-Hulin, M., Eisenberg, P.D., Mickiewicz, E., Celio, L., Pitt, P., Mita, M., Aaronson, N.K., Fowst, C., Arkhipov, A., di Salle, E., Polli, A. and Massimini, G. (2000) Activity of exemestane in metastatic breast cancer after failure of nonsteroidal aromatase inhibitors: a phase II trial. J Clin Oncol 18(11): 2234-2244.

10) Gianfilippo, B., Ornella, G., Marco, M. (2002) Sequential use of aromatase inactivators and inhibitors in advanced breast cancer. ASCO Abs\#238.

11) Chia, S., Gradishar, W., Mauriac, L., Bines, J., Amant, F., Federico, M., Fein, L., Romieu, G., Buzdar, A., Robertson, J.F., Brufsky, A., Possinger, K., Rennie, P., Sapunar, F., Lowe, E. and Piccart, M. (2008) Double-blind, randomized placebo controlled trial of fulvestrant compared with exemestane after prior nonsteroidal aromatase inhibitor therapy in postmenopausal women with hormone receptor-positive, advanced breast cancer: results from EFECT. J Clin Oncol 26(10): 1664-1670.

12) Yamamoto, Y., Masuda, N., Ohtake, T., Yamashita, H., Saji, S. Kimijima, I., Kasahara, Y., Ishikawa, T., Sawaki, M., Hozumi, Y. and Iwase, H. (2010) Clinical usefulness of high-dose toremifene in patients relapsed on treatment with an aromatase inhibitor. Breast Cancer. 17(4): 254-60.

13) Osborne, C.K., Zhao, H. and Fuqua, S.A.W. (2000) Selective estrogen receptor modulators: structure, function, and clinical use. J Clin Oncol 18: 3172-3186.

14) Asaishi, K., Tominaga, T., Abe, O., Izuo, M. and Nomura, Y. (1993) Efficacy and safety of high dose NK622 (Toremifene citrate) in tamoxifen failed patients with breast cancer. Jpn J Cancer Chemother 20(1): 91-99.

15) A, Gams. and Charles, L., Vogel. (1999) Toremifene (TOR): an active agent in the management of postmenopausal women with advanced breast cancer refractory to tamoxifen(TAM). ASCO Abs\#504. 